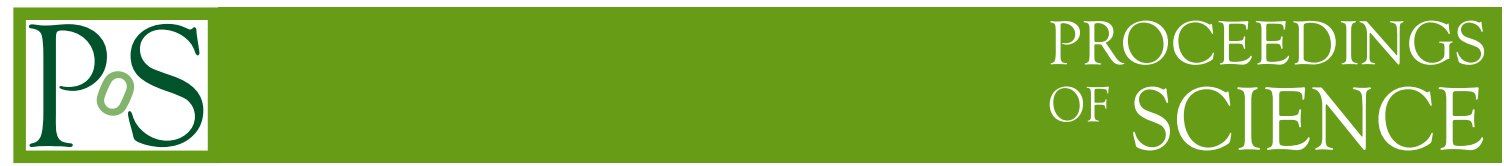

\title{
Schwinger-Dyson equations for manifestly gauge invariant correlators
}

\section{David Vercauteren*}

Universitat de València, Spain

E-mail: David.Vercauteren@UV.es

I present a formalism in which to study the correlators of gauge invariant operators using Schwinger-Dyson equations. All mention of gauge dependent fields, such as gluons and ghosts, is avoided, leaving only manifestly gauge invariant quantities appearing in the equations.

International Workshop on QCD Green's Functions, Confinement and Phenomenology, September 05-09, 2011

Trento Italy

\footnotetext{
* Speaker.
} 


\section{Introduction}

Schwinger-Dyson equations are a powerful analytic tool to study quantum field theories beyond perturbation theory. In writing down the equations, no approximations need to be made, which means that no physics is lost and that the equations contain, in principle, all physics - both perturbative and non-perturbative - exactly.

In a general quantum field theory, the equations follow from Stokes' theorem applied to the path integral:

$$
\int[d \phi] \frac{\delta}{\delta \phi(x)} e^{-S[\phi]+J \cdot \phi}=0 .
$$

From this master equation, the entire tower of Schwinger-Dyson equations can be written down by expansion in the source field $J(x)$ and equating the expansion coefficients. This leads to equations involving full (i.e. containing disconnected parts) $n$-point functions, and with some further manipulations they can be reduced to equations involving only connected or only 1PI Green's functions.

In the case of Yang-Mills theory (and, by extension, of QCD) the role of the field $\phi(x)$ is played by the gluon field $A_{\mu}^{a}(x)$. This means that the Schwinger-Dyson equations obtained this way will contain gluon propagators and gluon interaction vertices. But what, in the end, interests us most are not so much gluons but rather bound-states like glueballs (and, in QCD, hadrons), meaning that after solving the full tower of equations - supposing that we are smart enough to do so - we would still be facing the formidable task of putting all elements together to extract the physical quantities of interest. One can therefore wonder whether it would not be possible to write down a set of equations involving only glueballs, thus sidestepping at least one leg in the quest to a better understanding of Yang-Mills theory.

This leads us to the question of the gauge symmetry. The gluon field has a non-trivial gauge transform, which makes it rather tough to get rid of the gauge dependence when starting from something like (1.1) with the field considered the gluon field. Ideally, one would like to take the functional derivative in some gauge invariant way, such that only gauge invariant quantities show up in the resulting equations. These gauge invariant operators would then be interpreted as glueball operators, and the goal mentioned above would be reached.

It seems, however, that such an ambitious program does not come easily when working in the continuum. Work in that direction has been done in times past $[1,2,3,4]$, but it seems like the best results come when introducing a lattice regulator. This means that manifest Lorentz invariance will have to be sacrificed, and (hopefully) recovered when we are able to take the continuum limit. Furthermore, all such work done in the past (see, for example, [5, 6, 7]) focused on computing expectation values of Wilson loops, hoping to get a better understanding of confinement. In my work I aim for connected correlators, which should give more insight in the spectrum of the theory and in scattering amplitudes. 


\section{Construction of the equations}

\subsection{Schwinger-Dyson on the lattice}

Following [8], we define the functional derivative on the lattice as

$$
\delta_{U}^{a} Q(U, \ldots)=\lim _{\varepsilon \rightarrow 0} \frac{Q\left(e^{i \varepsilon \tau^{a} / 2} U, \ldots\right)-Q(U, \ldots)}{\varepsilon},
$$

where $\tau^{a}$ are the generators of the gauge group. Analogously, a right derivative can be defined. In practice, this operator works as

$$
\delta_{U}^{a} U=\frac{i \tau^{a}}{2} U
$$

and furthermore it obeys Leibniz's rule. When working on a Wilson loop containing the link matrix $U$, the derivative will "cut open" the loop and introduce a group generator. We will depict this as:

$$
\delta_{x, \mu}^{a} \underset{x}{\underset{\vec{\mu}}{\longrightarrow}}=\underbrace{\stackrel{a}{\longrightarrow \vec{\mu}}} .
$$

Due to the invariance of the Haar measure under gauge transformations, Stokes' theorem applies to the derivative thus defined:

$$
\int d U \delta_{U}^{a} Q(U, \ldots)=0 .
$$

In order to find a gauge invariant result from the lattice functional derivative, two derivatives are necessary. Using a well-known identity involving the gauge group generators, one finds in $\mathrm{SU}(N)$ gauge theory that

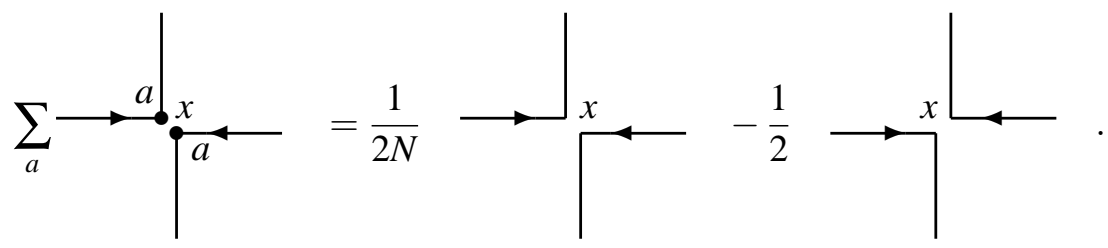

The sum over the gauge index closes the loops that were cut open (after eventual reconnection), thus once more resulting in gauge invariant quantities.

All this together bring us to the strategy to follow for writing down Schwinger-Dyson equations. The master equation is:

$$
\int[d U] \sum_{a} \delta_{x, \mu}^{a}\left(e^{-S[U]} Q[U] \delta_{x, \mu}^{a} R[U]\right)=0 .
$$

\subsection{Onepoint functions}

A first set of equations are the ones for the onepoint functions. As an example, let's consider the equation following from

$$
\int[d U] \sum_{a} \delta_{x, \mu}^{a}\left(e^{-S[U]} \delta_{x, \mu}^{a} P\right)=0
$$


where $P$ is a shorthand for the one-plaquette Wilson loop

$$
P=\frac{1}{N} \operatorname{tr}\left(U_{x, \mu} U_{x+\hat{\mu}, v} U_{x+\hat{v}, \mu}^{\dagger} U_{x, v}^{\dagger}\right) \quad(\mu \neq v) .
$$

Schematically, we find the equation

$$
-\frac{N^{2}-1}{2 N}\langle P\rangle+\frac{1}{2 g^{2}}-\frac{1}{2 g^{2}}\langle Q\rangle+\frac{1}{2 g^{2} N^{2}} \int d p f(p) \Delta_{P P}(p)=0,
$$

where $Q$ is a shorthand for the sum of several Wilson loop operators involving two plaquettes, $\Delta_{P P, i}(p)$ is the propagator or connected twopoint function of two one-plaquette Wilson loop operators in momentum space, and $f(p)$ is some function (also depending on the relative orientation of the two plaquettes in the propagator). This equation is equivalent to what one would find using the loop space formalism with a lattice regulator [4].

This equation can be drawn in Feynman diagrams, which looks like

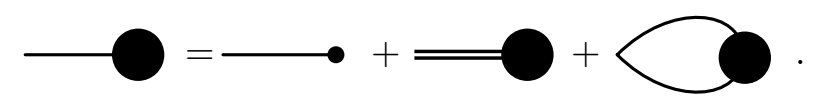

Here, a single line indicates the operator $P$, a double line indicates the operators included in $Q$, and a small dot at the end of the single line in the right-hand side is the contribution coming from the $1 / 2 g^{2}$ term in equation (2.9). The terms in the right-hand side can be interpreted as the tree-level contribution, a mixing term, and a one-loop correction.

Equations for vacuum expectation values of other operators than $P$ can be derived in a completely analogous way, with similar results.

\subsection{Propagators}

The equations for twopoint functions can be written down in a similar way, by simply adding a second Wilson loop operator at some other spacetime point. One should note, however, that at this point, it becomes of importance which of links of the first Wilson loop operator is chosen for the functional derivative. Different choices will lead to other equations, and the quickest way to nice first results seems to be by symmetrizing over all four links of the plaquette. Other ways are, of course, completely valid and may or may not lead to more insight about the theory.

The result for a pair of $P$ operators can be depicted as ${ }^{1}$

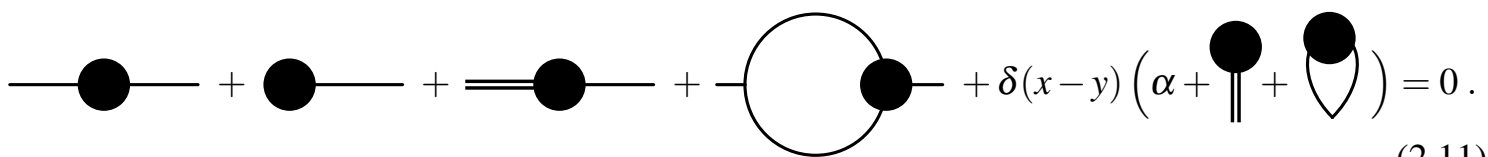

Here, $\alpha$ is a certain constant which is not important for my purposes. This is in terms of full Green's functions, i.e. including disconnected parts.

In order to study the connected Green's functions, we write all full Green's function as a sum of the connected and the disconnected parts. Terms including disconnected parts cancel each other upon using the Schwinger-Dyson equations for the onepoint functions. If we now define a tree-level inverse propagator as

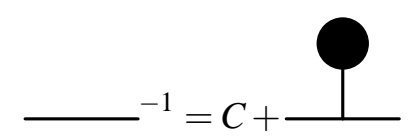

\footnotetext{
${ }^{1}$ I will not write down the full mathematical expressions, as they are quite unenlightening.
} 
with $C$ some constant following from the expressions, we can write down the Schwinger-Dyson equations in terms of 1PI Green's functions:

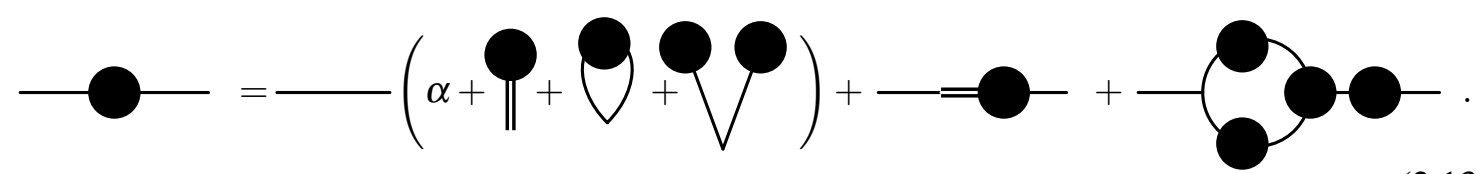

In order to get a better feeling for what is going on, let's look at the structure of the tree-level propagator. In the charge-conjugation even sector, it is (in momentum space) just a polynomial of the cosines of the components of the momentum vector. In the charge-conjugation odd sector, however, it turns out to have the denominator

$$
\sum_{\mu} 4 \sin ^{2} \frac{p_{\mu}}{2}-\frac{2 g^{2}\left(N^{2}-1\right)}{\langle P\rangle} .
$$

The first term is exactly the lattice equivalent of a momentum-squared term. The second term is a mass. At tree level, $\langle P\rangle$ is positive, meaning that the particle in question is tachyonic. ${ }^{2}$ This should, however, not yet worry us as both the mass and the condensate receive important contributions from the mixing terms.

Considering several more operators, one can take operator mixing into account, and one finds that the denominator of the propagator gets modified. Operator with larger extent than just one plaquette introduce higher powers of momenta, such that more zeros get generating, thus leading to a spectrum with ever more states, and also the charge-conjugation even sector starts to contain non-trivial physical content. I will say more about this in the next section.

\subsection{Interactions}

In the same vein, higher $n$-point functions can be investigated. However, there happen several things that are slightly out of the ordinary. For example, some of the terms found in the equation of the threepoint vertex are:

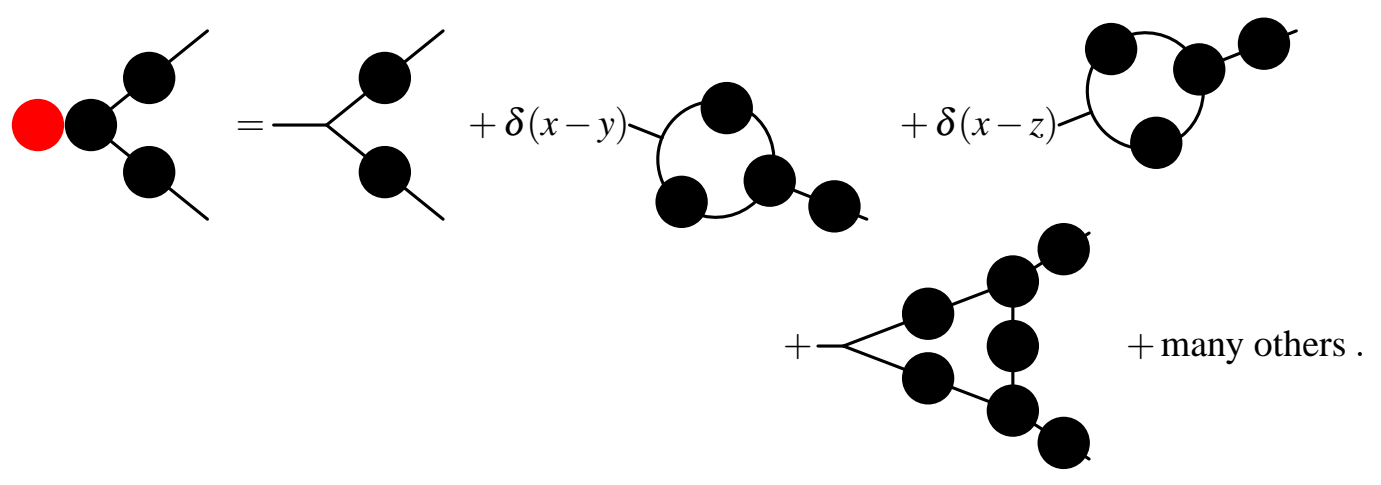

Here, the red blob in the left-hand side is the term between the brackets in equation (2.13). It is a polynomial of trigonometric functions of the momentum. The terms involving Kronecker-deltas do not appear in standard Schwinger-Dyson equations. I will say more about this in the next section.

\footnotetext{
${ }^{2}$ As we are working on the lattice, we use Euclidean spacetime.
} 


\section{Towards an expanded solution}

\subsection{Operator mixing and the strong-coupling expansion}

Ignore, for now, all diagrams containing loops. It is then very tempting to treat the treelevel term in equation (2.10) as a starting approximation and to then treat the operator mixing as a perturbation. This turns out to give the strong-coupling expansion, i.e. an expansion in ever increasing powers of $1 / g^{2} N$ or the inverse 't Hooft coupling. The propagator is a polynomial in the lattice momentum, and its degree increases as more powers of the inverse coupling are added. This means an ever more extensive spectrum is found.

On the one hand this looks very interesting, as it gives us a systematic way of computing physical observables. However, the coupling under consideration has not been renormalized in any way, and is actually the coupling defined at the cutoff - in this case the inverse lattice spacing. This means that the continuum limit consists in taking the coupling to zero, in which case a strongcoupling expansion is not very useful.

More research is underway in this direction.

\subsection{Loops and the large- $N$ expansion}

If we could, somehow, solve the theory at zero-loop level, we would then be in a position to compute corrections coming from loops. If we consider $g^{2} N$ to be a constant, then the loop expansion turns out to be an expansion in increasing powers of $1 / N^{2}$. Similar conclusions have already been found in the context of lattice Schwinger-Dyson equations in [5, 7], and in the context of continuum gauge invariant Schwinger-Dyson equations in [4].

When writing down this expansion, it seems that the delta terms in the threepoint function (2.15) "eat" propagators in loops. For example, the loop diagram in equation (2.13) contains two threepoint vertices - one bare one and one full one. When replacing the full one by its expression from (2.15), one of the propagators in the loop will disappear and a bare four-vertex arises. This means that the values of the bare vertices cannot be trivially read off from the relevant equations, as more contributions will appear when putting diagrams together.

\section{Open questions left}

One still open question concerns the meaning of the red blob in the equation (2.15). When plugging the threepoint vertex into other equations, the blob has to be inverted, which leads to a polynomial of the lattice moment appearing the denominator. This seems to mean that the theory might contain more particles than the one immediately found from the full zero-loop propagator.

Another open question concerns the form of the loop expansion. As the Schwinger-Dyson equations found in this work do not exactly have the standard form, solving them perturbatively will also lead to a loop expansion which is slightly out of the ordinary. This is related to the fact that the first leg of each $n$-point function in its own equation is always singled out as special. In standard Schwinger-Dyson equations this lack of symmetry disappears when writing down the perturbative expansion, which seems not to happen here.

Furthermore, due to the liberty of placing the functional derivative at different links along the main operator - as mentioned in paragraph 2.3 - there will be more equations than one gets in 
the standard Schwinger-Dyson approach. The question arises immediately whether and how these extra equations can be used to get further insights.

\section{References}

[1] Y. Nambu. QCD and the string model. Phys.Lett. B80, 372 (1979).

[2] E. Corrigan and B. Hasslacher. A functional equation for exponential loop integrals in gauge theories. Phys.Lett. B81, 181 (1979).

[3] J.-L. Gervais and A. Neveu. The quantum dual string wave functional in Yang-Mills theories. Phys.Lett. B80, 255 (1979).

[4] Yu. Makeenko and A. A. Migdal. Quantum chromodynamics as dynamics of loops. Nucl.Phys. B188, 269 (1981).

[5] T. Eguchi. Strings in U(N) lattice gauge theory. Phys.Lett. B87, 91 (1979).

[6] D. Weingarten. String equations for lattice gauge theories with quarks. Phys.Lett. B87, 97 (1979).

[7] D. Foerster. Yang-Mills theory - a string theory in disguise. Phys.Lett. B87, 87 (1979).

[8] W. Kerler. Schwinger-Dyson equations and currents in lattice gauge theory. Phys.Lett. B100, 267 (1981). 